\title{
Non-operative Management for Acute Appendicitis During the COVID-19 Pandemic Does Not Increase the Rate of Complications
}

\author{
Vishal K. Patel ${ }^{1} \cdot$ Kenny Ye ${ }^{2} \cdot$ Haejin $\mathrm{In}^{3} \cdot$ Meir H. Scheinfeld $^{4}$ (D) \\ Received: 8 August 2020 / Accepted: 17 October 2020 / Published online: 2 November 2020 \\ (C) 2020 The Society for Surgery of the Alimentary Tract
}

Keywords COVID-19 · COVID · Corona virus · Appendicitis · Appendectomy · Complications

\section{Introduction}

The COVID-19 pandemic has placed unprecedented stress on the healthcare system $\left.{ }^{1}\right]$. In response to government mandates and society recommendations, even emergency surgical procedures such as appendectomies have been curtailed, with non-surgical management used whenever possible [ $\left.{ }^{2,3}\right]$. The goal of our study was to determine whether changes in acute appendicitis management have altered the frequency of complications in these patients.

\section{Methods}

IRB approval was obtained for this retrospective cohort study performed at an urban tertiary care medical center during the COVID-19 pandemic which in our area extended from March 15 through May 31, 2020. The equivalent 77-day

This work has not been presented at a scientific meeting.

Supplementary Information The online version contains supplementary material available at https://doi.org/10.1007/s11605-02004844-8

\section{Meir H. Scheinfeld}

mscheinf@montefiore.org

Vishal K. Patel

vipate@montefiore.org

Kenny Ye

Kenny.Ye@einstein.yu.edu

Haejin In

hin@montefiore.org

1 Montefiore Medical Center, Department of Radiology, Albert Einstein College of Medicine, 111 East 210 Street, Bronx, NY 10467, USA period from 2019 was used for comparison. The imaging database was queried for all reports with the word "appendicitis." Imaging, operative notes, and pathology reports were used to classify cases as perforated or non-perforated appendicitis $\left[{ }^{4}\right]$. The EMR was reviewed for each case to extract the duration of symptoms prior to presentation, management, length of hospital stay (LOS), and complications within 45 days of presentation. Complications were divided into minor (Clavien-Dindo I and II) and major (Clavien-Dindo > II). Fisher exact tests were used to compare perforation rate, sex, baseline comorbidities, and non-operative treatment between years. Two sample $t$ tests were used to compare age. Linear regression models were used to compare the duration of symptoms and LOS with perforation as a covariate. A Mantel-Haenszel test was used to compare complications conditional on perforation. Statistical analyses were performed using R-software.
2 Department of Epidemiology \& Population Health, Department of Systems \& Computational Biology, Albert Einstein College of Medicine, 1300 Morris Park Avenue, Block BD \#310, Bronx, NY 10461, USA

3 Montefiore Medical Center, Department of Surgery, Department of Epidemiology and Population Health, Albert Einstein College of Medicine, 1300 Morris Park Avenue, Block BD \#112, Bronx, NY 10461, USA

4 Montefiore Medical Center, Department of Radiology, Division of Emergency Radiology, Albert Einstein College of Medicine, 111 East 210 Street, Bronx, NY 10467, USA 
Table 1 Comparison of clinical factors between the 2019 and 2020 cohorts

\begin{tabular}{|c|c|c|c|}
\hline & 2019 & 2020 & $p$ value \\
\hline \multicolumn{4}{|l|}{ Perforation } \\
\hline Acute appendicitis $(n)$ & 111 & 75 & \multirow[t]{2}{*}{0.17} \\
\hline Perforated acute appendicitis $(n, \%)$ & $23(21 \%)$ & $23(31 \%)$ & \\
\hline \multicolumn{4}{|c|}{ Days of symptoms prior to presentation (median $\pm \mathrm{IQR}$ ) } \\
\hline Perforated & $3 \pm 2.5$ & $3 \pm 1.5$ & \multirow[t]{2}{*}{0.47} \\
\hline Non-perforated & $1 \pm 1$ & $1 \pm 1$ & \\
\hline \multicolumn{4}{|l|}{ Treatment perforated $(n, \%)$} \\
\hline Non-operative & $4(17 \%)$ & $3(13 \%)$ & \multirow[t]{2}{*}{1.0} \\
\hline Other & $19(83 \%)$ & $20(87 \%)$ & \\
\hline \multicolumn{4}{|l|}{ Treatment non-perforated $(n, \%)$} \\
\hline Non-operative & $2(2 \%)$ & $21(40 \%)$ & \multirow[t]{2}{*}{$<0.001$} \\
\hline Other & $86(98 \%)$ & $31(60 \%)$ & \\
\hline \multicolumn{4}{|l|}{ Hospital length of stay (median \pm IQR) } \\
\hline Perforated & $4 \pm 6.5$ & $4 \pm 5$ & \multirow[t]{2}{*}{0.73} \\
\hline Non-perforated & $1 \pm 1$ & $1 \pm 2$ & \\
\hline
\end{tabular}

\section{Results}

There were 75 cases of acute appendicitis in 2020 compared to 111 in 2019 (Supplemental Figure 1, Supplemental Table 1). There was a trend towards increased perforated cases in 2020 $(23 / 75$ [31\%] in 2020 versus $23 / 111$ [21\%] in 2019) but was not statistically significant ( $p=0.17$, Table 1$)$. While nonoperative management for perforated cases was similar between 2020 and 2019 ( $p=1.0)$, non-operative management was significantly more common for non-perforated appendicitis cases in $2020(p<0.001$, Supplemental Table 2). Table 2 displays complications conditional on perforation and operative status. No difference was observed for complications between the 2 years $(p=0.85)$.
Of the 24 patients treated non-operatively in 2020,7 $(29 \%)$ underwent interval appendectomy at $84 \pm 39$ (mean $\pm \mathrm{SD}$ ) days after the initial presentation during 108-183 days of available follow-up. Three appendectomies were performed for persistent pain, two based on patient preference without new symptoms, one due to an asymptomatic appendicolith appearing on a follow-up exam, and one for definitive management of a previously perforated appendicitis.

\section{Discussion}

During the COVID-19 pandemic, the number of patients presenting with acute appendicitis markedly decreased compared to the prior year (32\% drop). We speculate that this was due to mild cases of appendicitis resolving spontaneously as patients' threshold for presenting to the hospital markedly increased during the pandemic. Spontaneous resolution of mild appendicitis has also been reported by Tankel et al. $\left[{ }^{5}\right]$. This suggests that we may be overtreating appendicitis, and efforts are needed to better distinguish patients who need surgical treatment versus those who do not.

This natural experiment corroborates other studies indicating that antibiotic treatment is an acceptable treatment alternative for non-perforated appendicitis, even during nonpandemic times $\left.{ }^{6}\right]$. Still, we also observed symptom-related interval appendectomies highlighting that such possibilities need to be discussed during informed consent and shared decision-making when deciding on the management of acute appendicitis.

Limitations of this study include single-center design and determination of complications based solely on representation at our institution.
Table 2 Complications during the 2019 and 2020 periods stratified by type of appendicitis, treatment performed ("nonoperative" indicating non-surgical treatment and "other" including appendectomy, elopement, and death), and complications

\begin{tabular}{lllll}
\hline & Treatment & Complications & $2019(n=111)$ & $2020(n=75)$ \\
\hline \multirow{2}{*}{ Perforated } & Non-operative & Major & 1 & 1 \\
& & Minor & 0 & 0 \\
& Other & None & 3 & 2 \\
& Major & 3 & 3 \\
& Minor & 2 & 3 \\
& Non-perforated & None & 14 & 14 \\
& Non-operative & Major & 0 & 0 \\
& & Minor & 0 & 0 \\
& & None & 2 & 21 \\
& Other & Major & 1 & 1 \\
& Minor & 3 & 0 \\
& & None & 82 & 30 \\
\hline
\end{tabular}


In conclusion, despite marked changes in the healthcare system during the COVID-19 pandemic and changes in appendicitis management, the complication rate remained the same compared to the pre-pandemic period despite a sharp spike in non-operative management.

Authors' contributions Protocol/project development: Patel, Ye, Scheinfeld

Data collection or management: Patel, Scheinfeld

Data analysis: Patel, Ye, In, Scheinfeld

Manuscript writing/editing: Patel, Ye, In, Scheinfeld

Final approval: Patel, Ye, In, Scheinfeld

\section{Compliance with ethical standards}

Conflict of interest The authors declare that they have no conflict of interest.

\section{References}

1. Uppal A, Silvestri DM, Siegler M, et al. Critical Care And Emergency Department Response At The Epicenter Of The COVID-19 Pandemic [published online ahead of print, 2020
Jun 11]. Health Aff (Millwood). 2020;101377hlthaff202000901. https://doi.org/10.1377/hlthaff.2020.00901

2. Collard M, Lakkis Z, Loriau J, et al. Antibiotics alone as an alternative to appendectomy for uncomplicated acute appendicitis in adults: Changes in treatment modalities related to the COVID-19 health crisis. J Visc Surg. 2020;157(3S1):S33-S42. https://doi.org/10. 1016/j.jviscsurg.2020.04.014

3. Søreide K, Hallet J, Matthews JB, et al. Immediate and long-term impact of the COVID-19 pandemic on delivery of surgical services [published online ahead of print, 2020 Apr 30]. Br J Surg. 2020;https://doi.org/10.1002/bjs.11670.

4. Foley TA, Earnest F 4th, Nathan MA, Hough DM, Schiller HJ, Hoskin TL. Differentiation of nonperforated from perforated appendicitis: accuracy of CT diagnosis and relationship of CT findings to length of hospital stay. Radiology. 2005;235(1):89-96. https://doi. org/10.1148/radiol.2351040310

5. Tankel J, Keinan A, Blich O, et al. The Decreasing Incidence of Acute Appendicitis During COVID-19: A Retrospective Multicentre Study. World J Surg. 2020;44(8):2458-2463. https://doi.org/ 10.1007/s00268-020-05599-8

6. Salminen P, Tuominen R, Paajanen H, et al. Five-Year Follow-up of Antibiotic Therapy for Uncomplicated Acute Appendicitis in the APPAC Randomized Clinical Trial. JAMA. 2018;320(12):12591265. https://doi.org/10.1001/jama.2018.13201

Publisher's Note Springer Nature remains neutral with regard to jurisdictional claims in published maps and institutional affiliations. 